\title{
Mammakarzinom - im Spannungsfeld von Medizin und Politik
}

I m Jahr 2003 sind nach Schätzungen 47500 Neuerkrankungen an Mammakarzinomen aufgetreten, 360000 Frauen sind nach ihrer Therapie aktuell ohne Rezidiv oder Metastasen, 55000 Frauen befinden sich in der metastasierten Situation, und 16600 Frauen sind am Mammakarzinom verstorben. Diese Zahlen und die Effekthascherei durch Pressemitteilungen, laut derer die Qualität der Behandlung in Deutschland unter dem europäischen Standard liegt, haben das Mammakarzinom ins Zentrum der politischen Diskussion gerückt. Sachlich sind die Argumente über die höhere Mortalität - insbesondere im Vergleich zu den USA und Großbritannien - nicht nachvollziehbar, da in Deutschland keine Dokumentationspflicht besteht und so keine vergleichbaren flächendeckenden Daten zum Resultat der Mammakarzinomtherapie vorliegen. Die gut dokumentierten prospektiven Untersuchungen deutscher Studiengruppen befinden sich jedoch auch international auf ausgesprochen hohem Niveau. Dies als Hintergrund für die aktuellen Diskussionen um neu zu implementierende Versorgungsstrukturen bzw. -qualität.

Zentraler Bestandteil der aktuellen Versorgungsstruktur von Brustkrebspatientinnen ist das DiseaseManagement-Programm (DMP) „Mammakarzinom“ eines von derzeit zwei in Deutschland zu implementierenden Programmen für chronisch Erkrankte. Über den Risiko-Strukturausgleich sollen solche Programme einen finanziellen Ausgleich zwischen den Kassen schaffen. Mit dem Einsteigen in ein DMP erhält der zentrale Koordinator eine Steuerungsfunktion, die in den nachfolgenden Jahren die Patientin kontinuierlich im Verlauf der Erkrankung begleiten bzw. notwendige diagnostische und therapeutische Maßnahmen koordinieren soll.

Die neben dem zentralen Koordinator notwendigen Strukturen sind die so genannten Brustkrebszentren (DMP-Zentren). Ziel ist eine - flächendeckende - Zertifizierung solcher spezieller Zentren nach den Kriterien der Deutschen Krebsgesellschaft bzw. der Deutschen Gesellschaft für Senologie. Der Anforderungskatalog beider Fachgesellschaften definiert Grundsätze zur Struktur, Qualität und Dokumentation. Dazu zählt auch die Teilnahme an bzw. die Durchführung von klinischen Studien. Es besteht eine hohe Übereinstimmung dieser Normen mit den internationalen EUSOMA-Kriterien („European Society of Mastology“), sodass der deutsche Qualitätsstandard dem europäischen entspricht.

Handlungsgrundlage im DMP und den Brustkrebszentren sind Standards, Leitlinien oder Richtlinien für die einzelnen interdisziplinären Versorgungseinheiten bzw. -schritte. Unklar ist, wie die Honorierung der Leistung im Rahmen des DMP bzw. in den DMP-definierten Kliniken erfolgt, da zeitgleich zu den DMPs auch die DRGs („diagnosis related groups“) eingeführt wurden.
Unter anderem bildet der Leistungskatalog der DRGs die plastisch rekonstruktiven Eingriffe nicht suffizient ab. Diese sind jedoch unabdingbar für die regelgerechte Versorgung einer Brustkrebspatientin, um deren körperliche Integrität wiederherzustellen. Gleiches gilt für die medikamentöse und die kombinierte medikamentösestrahlentherapeutische Therapie. Zentraler Punkt zur Evaluierung bzw. zur Verbesserung der Qualität durch die neu implementierten Versorgungsstrukturen ist die Dokumentation und deren Auswertung in entsprechenden umfassenden Programmen. Problematisch hierbei ist, dass teilweise eine parallel laufende Dokumentation - zum Beispiel in den klinischen bzw. epidemiologischen Krebsregistern und im DMP - erfolgt.

Das Gesundheitssystem steht vor einer deutlichen Wandlung mit Fokussierung spezifischer Krankheitsbilder auf zentralistische Strukturen. Ob sich damit die Qualität der Versorgung verbessern lässt, muss durch Ergebnisdokumentation und Qualitätsüberprüfung nachgewiesen werden. Dieses ist umso wichtiger, da mit der Europäisierung und damit der Vereinheitlichung der Strukturen in den Gesundheitssystemen deutsche Versorgungsstrukturen konkurrenzfähig bleiben.

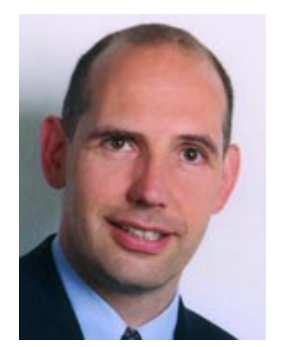

Prof. Dr. M.W. Beckmann, Erlangen

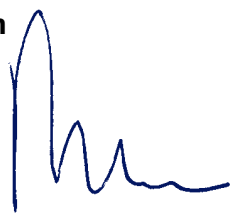

